\title{
Increased levels of angiogenic factors in microvascular angina
}

Elmira Roshani As1 ${ }^{1}$, Yousef Rasmi ${ }^{1,2}$, Mohammad-Hasan Khadem-Ansari ${ }^{1}$, MirHossein Seyed-Mohammadzad ${ }^{3}$, Alireza Rostamzadeh ${ }^{3}$, Fereshteh Ghaffari ${ }^{1}$, Narmin Mokarizadeh $^{1}$

1) Department of Biochemistry, School of Medicine, Urmia University of Medical Sciences, Urmia, Iran

2) Cellular and Molecular Research Center, Urmia University of Medical Sciences, Urmia, Iran

3) Department of Cardiology, School of Medicine, Urmia University of Medical Sciences, Urmia, Iran

DOI: $10.15386 /$ cjmed-1101

Manuscript received: 03.07.2018 Received in revised form: 16.09 .2018 Accepted: 27.09.2018

Address for correspondence: yrasmi@gmail.com

\begin{abstract}
Background. Recent studies have suggested that angiogenic factors may affect vascular endothelial integrity. On the other hand, endothelial dysfunction is the main pathological mechanism in microvascular angina (MVA) or cardiac syndrome X. Therefore, we aimed to determine the levels of angiogenic factors in MVA patients. In addition, we investigated the effects of metoprolol, as a beta blocker agent, on the serum levels of these factors.

Methods. Thirty patients with MVA (17 female/13 male; mean age: $55.53 \pm 9.18$ years) and twenty healthy controls ( 14 female/6 male; mean age: $51.40 \pm 9.16$ years) were enrolled.

The serum amounts of angiopoietin-1 (Ang-1), angiopoietin-2 (Ang-2) and tyrosine kinase-2 receptor (Tie-2) were measured in healthy controls, MVA patients at baseline and after metoprolol therapy ( $25 \mathrm{mg}$ for one month) by enzyme-linked immunosorbent assay.
\end{abstract}

Results. The levels of Ang-2 and Tie-2 were significantly higher in MVA patients at baseline in comparison with controls (Ang-2: $277.02 \pm 186.08$ vs. $164.46 \pm 49.83 \mathrm{ng} / \mathrm{l}$, $\mathrm{P}=0.011$; Tie- 2 : $28.97 \pm 18.85$ vs. $14.90 \pm 4.05 \mathrm{ng} / \mathrm{ml}, \mathrm{P}=0.002$; respectively). But this difference in the Ang-1 levels was not significant $(\mathrm{P}=0.829)$. Additionally, the levels of angiogenic factors in MVA patients after metoprolol therapy were not significantly changed in comparison with the baseline status $(\mathrm{P}>0.05)$.

Conclusion. Our results considered a possible role for angiogenic factors in the pathophysiology of MVA, which need further investigation for elucidation. In addition, this study has not showed an effective role for metoprolol in changing the angiogenic factors levels as a therapeutic agent in MVA.

Keywords: angiopoietin, cardiac syndrome X, metoprolol, tyrosine kinase

\section{Introduction}

Cardiovascular disease, a common consequence of coronary arteries obstruction, is one of the most important causes of death and disability, worldwide [1]. Up to $40 \%$ of patients suffering from chest discomfort characteristic of angina and undergoing invasive angiography present no sign of obstructive atherosclerosis [2,3]. Exclusion of noncardiac diagnoses such as gastrointestinal or psychiatric disorders, as well as the presence of four important features including angina-like chest pain, ST segment depression during angina $(>1 \mathrm{~mm})$, normal epicardial coronary arteries at angiography, and no sign of spontaneous or inducible epicardial coronary artery spasm on ergonovine or acetylcholine provocation, are critical hallmarks for cardiac syndrome X (CSX), a historic term for microvascular angina (MVA) diagnosis [3-5]. While various plausible mechanisms are introduced for chest pain in MVA patients, the primary dysfunction of the small coronary arteries ( $<500 \mathrm{~mm}$ in diameter), coronary microvascular dysfunction, as an intricate and incompletely understood condition, is suggested to have a substantial etiological role in the pathogenesis of MVA [6,7].

Angiogenesis is a sophisticated process, which is operated by the highly regulated signaling events among angiogenic factors [8]. Vascular endothelial growth factor (VEGF), tyrosine kinase-2 receptor (Tie-2) and its ligands, angiopoietin-1 (Ang-1) and angiopoietin-2 (Ang-2), which are primarily expressed on endothelial cells 
and early hematopoietic cells [9], have been demonstrated to play a substantial functions in developmental blood vessel formation and modulation of hypoxia-induced tissue angiogenesis [10]. These factors can stabilize endothelial interactions with their supporting cells via promoting endothelial cells survival; reduce vascular permeability by slight effect on endothelial cells proliferation [11]. On the other hand, Ang-2 may destabilize the vasculature and results in vessel regression. All of these effects are mediated by the binding of Ang-1 and Ang-2 to their receptor termed the ECs- specific tyrosine kinase receptor Tie-2 [12]. Ang/Tie-2 signaling pathway has central role in regulating physiologic and pathologic angiogenesis, and it is also a specific therapeutic target for the treatment of vascular disease and cancer. Alteration in the levels of Ang1, Ang-2 and Tie-2 were reported in some cardiovascular diseases such as acute coronary syndrome, congestive heart failure, myocardial infraction, and diabetes-related unstable angina pectoris [13-19]. However, to date the MVA-related alterations in the levels of Ang-1, Ang-2 or the Tie-2 receptor expression, as well as the effects of treatment with metoprolol, as the first line agent for the treatment of MVA, have not been defined in patients with this cardiovascular disease. Characterization of changes in endogenous Ang1, Ang-2 and Te-2 expression induced by MVA may be essential for the elucidation of the role of this new family of angiogenic mediators in the pathogenesis of MVA, as well as possible targeting of these proteins as a therapeutic strategy in improving endothelial dysfunction. In this study, we aimed to investigate the angiogenesis status in patients with MVA by measuring the levels of Ang-1, Ang-2 and Tie- 2 and then assess the effects of metoprolol therapy on these parameters.

\section{Materials and methods Study participants}

In this study we enrolled MVA patients and apparently healthy controls. The case group consisted of 30 patients (13 male and 17 female, mean age: $55.53 \pm 9.18$ years) who referred to our institution for suspected coronary artery disease. The entry criteria were typical angina- like chest pain, a normal 12-lead ECG at rest, a positive exercise ECG stress test response ( $>1.5 \mathrm{mV}$ ST-segment depression at 60 times after the $\mathrm{j}$ point in 2 contiguous leads that for $>1$ minute) and normal coronary angiogram. Non-cardiac causes of chest pain and other dysfunctions such as left ventricular dysfunction, myocardial infarction, valvular heart disease and myocardial hypertrophy on M- and Bmode echocardiography, pericardial disease and psychiatric disorders, other systemic disease such as diabetes mellitus, renal, hepatic and pulmonary disorders, acute and chronic infection and hypertension were not included in this study. All medical history was collected before physical examination, including complaints, existence of other diseases, risk factors and cardiac and non-cardiac medications. Additionally, the control group consisted of 20 ( 6 male and 14 female, mean age: $51.40 \pm 9.16$ years) apparently healthy volunteers. None of the controls had a previous history of chest pain, diabetes and hypertension, acute and chronic diseases. Also they did not take any kind of cardiac or non-cardiac medication and dietary supplements. The study was approved by the University Medical Ethics Committee and informed consent was obtained from all subjects.

A $5 \mathrm{ml}$ blood sample was obtained from each subject and centrifuged at $300 \times \mathrm{g}$ for 20 minutes. Serum was aliquoted and stored at $-80{ }^{\circ} \mathrm{C}$ until analysis. Then metoprolol, 25 $\mathrm{mg}$ orally, was given daily to patients and maintained for 4 weeks. After mentioned time, we collected serum samples in same conditions and stored for biochemical analysis.

\section{Biochemical analysis}

Serum samples were used for the measuring the angiogenic markers levels. For Ang-1 measuring, we used sandwich ELISA method and commercial kit (Shanghai crystal day Biotech., China); according to the manufacturer's instruction sensitivity was $5.49 \mathrm{pg} / \mathrm{ml}$. We used the same method for Ang-2 (Shanghai crystal day Biotech., China; sensitivity: $2.49 \mathrm{ng} / \mathrm{l}$ ) and Tie-2 receptor (Shanghai crystal day Biotech., China; sensitivity: 0.23ng/ $\mathrm{ml})$. All three markers were measured in three groups including controls, MVA patients at baseline and after metoprolol therapy.

\section{Statistical analysis}

The data were analyzed in SPSS software version 21. Quantitative parameters including age, BMI, blood pressure and angiogenic markers were shown as mean \pm standard deviation (SD) and for analyzing qualitative parameters, we used proportion. Kolmogorov-Smirnov test was used to determine the parametric distribution of the variable. For comparison of quantitative parameters, $\mathrm{t}$-test, and for comparison of the parameters levels after and before treatment, Wilcoxon test (paired t-test) were used. In addition, qualitative data was interpreted on the basis of Chi square test. A p-value less than 0.05 was accepted as statistically significant.

\section{Results}

The main demographic features of MVA patient and control groups are shown in table I. A comparison of angiogenic markers levels between controls and MVA patients before treatment was first part of this study. In MVA patients, the levels of Ang-2 and Tie-2 were significantly higher than controls $(\mathrm{P}=0.011$ and $\mathrm{P}=0.002$, respectively). But Ang-1 levels between these groups were not significant $(\mathrm{p}=0.829$, Table II).

The second part of study was the assessment of angiogenic markers in MVA patients before and after treatment with metoprolol. The levels of Ang-1, Ang-2 and Tie-2 were not significantly different between groups $(\mathrm{P}=$ $0.711, \mathrm{P}=0.229, \mathrm{P}=0.216$; respectively, Table II). 
Table I. Main demographic characteristics of microvascular angina (MVA) and control groups

$\begin{array}{llll} & \text { Control group } & \text { MVA group } & \text { Pvalue } \\ \text { Gender (male/female) } & 6 / 14 & 13 / 17 & 1.000 \\ \text { Age (years) } & 51.40 \pm 9.16 & 55.53 \pm 9.18 & 0.161 \\ \text { Body mass index (kg/m2) } & 26.00 \pm 1.88 & 28.60 \pm 5.83 & 0.061 \\ \text { Systolic blood pressure (mm Hg) } & 115 \pm 8.27 & 123 \pm 19.50 & 0.112 \\ \text { Diastolic blood pressure (mmHg) } & 71.50 \pm 8.12 & 72.50 \pm 10.56 & 0.722\end{array}$

Table II. Angiogenic factors levels in controls and MVA patients at baseline and after metoprolol therapy

\begin{tabular}{l|l|l|l|l|l} 
Angiogenic factors & Controls & MVA at baseline & MVA after treatment & P1 & P2 \\
Angiopoetin-1 (pg/ml) & $801.62 \pm 392.28$ & $780.63 \pm 240.23$ & $749.61 \pm 349.42$ & 0.829 & 0.711 \\
Angiopoetin-2 (ng/l) & $164.46 \pm 49.83$ & $277.02 \pm 186.08$ & $204.77 \pm 95.00$ & 0.011 & 0.229 \\
Tyrosine kinase-2 receptor (Tie-2) $(\mathrm{ng} / \mathrm{ml})$ & $14.90 \pm 4.05$ & $28.97 \pm 18.85$ & $21.14 \pm 11.8$ & 0.002 & 0.216
\end{tabular}

P1: controls and baseline MVA

P2: Baseline MVA and after metoprolol therapy

\section{Discussion}

Endothelial dysfunction, which is characterized by imbalance between vaso-dilating and vaso-constricting substances generated by the endothelium, is the most significant mechanism involved in the pathogenesis of MVA [20]. Therefore, the exact attribution of endothelial dysfunction and the factors that influence endothelial function in MVA is a subject of great interest for researchers in this field. In our previous studies, we investigated the various aspects of this subject and assessed different factors that may be involved in the pathogenesis of the MVA and we obtained attractive results, even offered a hypothesis in this area [21-24]. As a follow-up of our research chain about MVA, in this study we have addressed the involvement of angiogenesis and the levels of angiogenic factors in MVA. Our results showed that the levels of two important angiogenic factors including Ang-2, but not Ang-1, and their receptor levels, Tie-2, were significantly increased in patients with MVA compared with healthy subjects. On the other hand, we administrated metoprolol to MVA group and observed that angiogenic factors levels were not significantly changed.

Accumulating previous studies had evaluated the involvement of angiogenic factors in the pathogenesis of various cardiovascular diseases. For instance, Lee et al." measured plasma Ang-1, Ang-2, and Tie-2 alongside VEGF in patients suffering from acute chronic syndrome (ACS) and compared with patients with stable angina and healthy controls. They found that Ang-2, Tie-2 and VEGF, but not Ang-1 levels, were higher in patients with ACS compared to controls [14]. In another study, Chen, et al. investigated the association of Ang-1 and Ang-2 levels with heart failure in patients with acute myocardial infarction. They found that the levels of Ang-1 and Ang-2 were increased significantly in patients with heart failure as compared with patients without heart failure [25]. In a rat model of myocardial infarction, Sandhu et al. showed a predominant role for
Ang-2 in the angiogenic response to myocardial infarction. Myocardial ischemia induced in rats by left coronary artery ligation led to a considerable increase in Ang-2 expression and a reciprocal decrease in Ang-1 [15]. Chong et al. in another study showed abnormal levels of Ang-2 and Tie-2, but normal Ang-1 levels in patients with congestive heart disease [13]. As mentioned before, impaired relaxation or enhanced sensitivity to vasoconstriction in the intramural arterioles and pre arterioles in patients with MVA leads to significant impairment in the myocardial blood supply and episodes of ischemia. This situation may be a reasonable explanation for the increased angiogenic signaling in MVA patients, in accordance to other cardiovascular diseases, in which coronary artery obstruction increases angiogenesis in order to recover from ischemia induced myocardial damages. The correlation of angiogenic factors such as Ang1, Ang-2, with endothelial dysfunction was reported in some studies. For example, Lim et al. measured Ang-1, Ang-2, and VEGF alongside other endothelial dysfunction markers in 94 patients with diabetes. They documented that plasma Ang-2 and VEGF, but not Ang-1 levels, were significantly increased in diabetes patients regardless of cardiovascular disease. They found a significant relation between endothelial dysfunction markers such as vWF levels [26]. In summary, we have shown that angiogenesis and increase in angiogenic factors is related to endothelial dysfunction and probably changes in angiogenesis activation in heart muscle, as a result of endothelial dysfunction, may play a possible role in the pathophysiology of MVA patients.

The lack of special definition for MVA, different pathogenic mechanism responsible for the condition, as well as the varied diagnostic criteria used by different investigators have contributed to complications in managing these patients [27]. Therefore, management of these patients is a complex challenge. Undoubtedly, beta blockers are the first line agents for treatment of MVA patients [3]. Many studies evaluated the effectiveness 
of this blocker in MVA patients [28]. In our previous studies, we showed that metoprolol efficiently improved the endothelial function in patients with MVA through significantly decreasing the endothelin-1, E-selectin, and vascular cell adhesion molecule-1 (VCAM-1) levels, as specific markers of endothelial dysfunction [7]. In other study, Sen et al. assessed the effects of metoprolol and nebivolol on endothelial function in MVA patients. They documented that nebivolol had better effect on endothelial function [29]. Moreover, Bank et al. evaluated the effects of metoprolol and carvedilol on endothelial function in diabetic patients. They showed that carvedilol had better effects than metoprolol on endothelial function [30]. Zhang et al. investigated the effects of metoprolol on angiogenesis and expression of VEGF factor in 36 rats with ischemic heart muscle. They reported that metoprolol intervened in the induction of angiogenesis in rats with ischemic heart muscle [31]. However, we did not observe any beneficial effects of metoprolol on the angiogenic factors in MVA patients. Therefore, metoprolol may exert its therapeutic action through other mechanisms such as improvement of endothelial function, not angiogenesis in MVA.

The main limitation of the present study was the small number of patients with MVA. It will be very useful to see the effect of metoprolol therapies on the other angiogenesis-related factors such as vascular endothelial growth factor. Therefore, large and long-term follow-up studies are needed with patients with MVA after metoprolol administration. Other limitation was the lack of a measure for endothelial dysfunction in the subjects investigated and its correlation with angiogenesis.

\section{Conclusion}

In present study, Ang-2 and Tie-2 levels were not statistically significantly changed after one month treatment with metoprolol in MVA patients compared to patients at baseline. That may be due to the small population, short duration of treatment or small dose of drug. We showed abnormal levels for Ang-2 and Tie-2 in MVA patients. These abnormalities may be considered as a possible relation of these angiogenic factors to the pathophysiology of MVA, which need further investigation for elucidation. In addition, in our study, because of slight reduction in angiogenic factors after treatment with metoprolol (25 $\mathrm{mg}$ ) during one month, we hypothesize that metoprolol has not conspicuous effect on levels of angiogenic factors in MVA patients. Our study supports further research into the complex interactions between Ang-1 and Ang-2 at the Tie2 receptor, and into their functional relevance in vivo.

\section{References}

1. Liang KW, Sheu WH, Lee WJ, Lee WL, Pan HC, Lee IT, et al. Post-challenge insulin concentration is useful for differentiating between coronary artery disease and cardiac syndrome $\mathrm{X}$ in subjects without known diabetes mellitus. Diabetol Metab Syndr.
2017;9:10. doi: 10.1186/s13098-017-0209-1.

2. Rosano GMC, Tousoulis D, McFadden E, Clarke J, Davies GJ, Kaski JC. Effects of neuropeptide Y on coronary artery vasomotion in patients with microvascular angina. Int J Cardiol. 2017;238:123-127.

3. Marinescu MA, Löffler AI, Ouellette M, Smith L, Kramer CM, Bourque JM. Coronary microvascular dysfunction, microvascular angina, and treatment strategies. JACC Cardiovasc Imaging. 2015;8:210-220.

4. Löffler AI, Bourque JM. Coronary microvascular dysfunction, microvascular angina, and management. Curr Cardiol Rep. 2016;18:1.

5. Chaudhary I, Kaski J. Cardiac syndrome X: Angina pectoris with normal coronary arteries. UpToDate. Waltham, MA. 2009.

6. Brainin P, Frestad D, Prescott E. The prognostic value of coronary endothelial and microvascular dysfunction in subjects with normal or non-obstructive coronary artery disease: A systematic review and meta-analysis. Int J Cardiol. 2018;254:1-9. 7. Majidinia M, Rasmi Y, Khadem Ansari MH, SeyedMohammadzad M, Saboory E, Shirpoor A. Metoprolol improves endothelial function in patients with cardiac syndrome X. Iran J Pharm Res. 2016;15:561-566.

8. Deveza L, Choi J, Yang F. Therapeutic angiogenesis for treating cardiovascular diseases. Theranostics. 2012;2:801-814.

9. Linares PM, Chaparro M, Gisbert JP. Angiopoietins in inflammation and their implication in the development of inflammatory bowel disease. A review. J Crohns Colitis. 2014;8:183-190.

10. Eklund L, Kangas J, Saharinen P. Angiopoietin-Tie signalling in the cardiovascular and lymphatic systems. Clin Sci (Lond). 2017;131:87-103.

11. Kiss EA, Saharinen P. Anti-angiogenic Targets: Angiopoietin and Angiopoietin-Receptors. Tumor Angiogenesis: Springer; 2018. pp. 1-24.

12. Saharinen P, Eklund L, Alitalo K. Therapeutic targeting of the angiopoietin-TIE pathway. Nat Rev Drug Discov. 2017;16:635661.

13. Chong AY, Caine GJ, Freestone B, Blann AD, Lip GY. Plasma angiopoietin-1, angiopoietin-2, and angiopoietin receptor tie-2 levels in congestive heart failure. J Am Coll Cardiol. 2004;43:423428.

14. Lee KW, Lip GY, Blann AD. Plasma angiopoietin-1, angiopoietin-2, angiopoietin receptor tie-2, and vascular endothelial growth factor levels in acute coronary syndromes. Circulation. 2004;110:2355-2360.

15. Sandhu R, Teichert-Kuliszewska K, Nag S, Proteau G, Robb MJ, Campbell AI, et al. Reciprocal regulation of angiopoietin-1 and angiopoietin-2 following myocardial infarction in the rat. Cardiovasc Res. 2004;64:115-124.

16. Gui C, Li SK, Nong QL, Du F, Zhu LG, Zeng ZY. Changes of serum angiogenic factors concentrations in patients with diabetes and unstable angina pectoris. Cardiovasc Diabetol. 2013;12:34. . 17. Lorbeer R, Baumeister SE, Dörr M, Felix SB, Nauck M, Grotevendt A, et al. Angiopoietin-2, its soluble receptor Tie-2 and subclinical cardiovascular disease in a population-based sample. Heart. 2015; 101:178-184.

18. Tsai YC, Lee CS, Chiu YW, Kuo HT, Lee SC, Hwang SJ, et al. Angiopoietin-2, Angiopoietin-1 and subclinical cardiovascular disease in chronic kidney disease. Sci Rep. 2016;6: 39400.

19. Tsai YC, Lee CS, Chiu YW, Kuo HT, Lee SC, Hwang SJ, et al. Angiopoietin-2 as a prognostic biomarker of major adverse 
cardiovascular events and all-cause mortality in chronic kidney disease. PLoS One. 2015;10:e0135181.

20. Arroyo-Espliguero R, Kaski JC. Microvascular dysfunction in cardiac syndrome $\mathrm{X}$ : the role of inflammation. CMAJ. 2006; $174: 1833$.

21. Rasmi Y, Mehraban K, Sadreddini M, Zeynalzadeh J, Majidinia M, Seyyed-Mohammadzad M, et al. Lack of significant association between Helicobacter pylori infection and homocysteine levels in patients with cardiac syndrome X. Cardiol J. 2012;19:466-469.

22. Rasmi Y, Raeisi S. Possible role of Helicobacter pylori infection via microvascular dysfunction in cardiac syndrome $\mathrm{X}$. Cardiol J. 2009;16:585-587.

23. Rasmi Y, Raeisi S, Seyyed Mohammadzad MH. Association of inflammation and cytotoxin-associated gene a positive strains of helicobacter pylori in cardiac syndrome X. Helicobacter. 2012;17:116-120.

24. Rasmi Y, Seyyed-Mohammadzad MH. Frequency of Helicobacter pylori and cytotoxine associated gene A antibodies in patients with cardiac syndrome X. J Cardiovasc Dis Res. 2012;3:19-21.

25. Chen S, Guo L, Chen B, Sun L, Cui M. Association of serum angiopoietin-1, angiopoietin-2 and angiopoietin-2 to angiopoietin-1 ratio with heart failure in patients with acute myocardial infarction. Exp Ther Med. 2013;5(3):937-941.

26. Lim HS, Blann AD, Chong AY, Freestone B, Lip GY. Plasma vascular endothelial growth factor, angiopoietin-1, and angiopoietin-2 in diabetes: implications for cardiovascular risk and effects of multifactorial intervention. Diabetes Care. 2004;27:2918-2924.

27. Agrawal S, Mehta PK, Bairey Merz CN. Cardiac syndrome X: update. Heart Fail Clin. 2016;12:141-156.

28. Kaski JC, Valenzuela Garcia LF. Therapeutic options for the management of patients with cardiac syndrome X. Eur Heart J. 2001;22:283-293.

29. Sen N, Tavil Y, Erdamar H, Yazici HU, Cakir E, Akgül EO, et al. Nebivolol therapy improves endothelial function and increases exercise tolerance in patients with cardiac syndrome X. Anadolu Kardiyol Derg. 2009;9:371-379.

30. Bank AJ, Kelly AS, Thelen AM, Kaiser DR, GonzalezCampoy JM. Effects of carvedilol versus metoprolol on endothelial function and oxidative stress in patients with type 2 diabetes mellitus. Am J Hypertens. 2007;20:777-783.

31. Zhang R, Liu X. The Effects of Metoprolol on angiogenesis and expresssion of VEGF in ischemic myocardium of rats. Journal of Guiyang Medical College. 2008;1:22-25. 